OPEN ACCESS

Edited by:

Desheng Liang,

Central South University, China

Reviewed by:

Qifei Li,

Boston Children's Hospital and

Harvard Medical School,

United States

Weimin Bi,

Baylor College of Medicine,

United States

*Correspondence:

Huijun Wang

huijunwang@fudan.edu.cn

Ying Huang

yhuang815@163.com

${ }^{\dagger}$ These authors have contributed equally to this work

Specialty section:

This article was submitted to

Genetics of Common and Rare

Diseases,

a section of the journal

Frontiers in Genetics

Received: 16 February 2021

Accepted: 12 April 2021

Published: 06 May 2021

Citation:

Qian Y, Wu B, Liu R, Lu Y, Zhang P,

Shao C, Huang $Y$ and Wang $H$ (2021)

Case Report: Complete Maternal

Uniparental Isodisomy of

Chromosome 5 (iUPD(5)mat) With

PCSK1 Nonsense Variant in an Infant

With Recurrent Diarrhea.

Front. Genet. 12:668326.

doi: 10.3389/fgene.2021.668326

\section{Case Report: Complete Maternal Uniparental Isodisomy of Chromosome 5 (iUPD(5)mat) With PCSK1 Nonsense Variant in an Infant With Recurrent Diarrhea}

\author{
Yanyan Qian ${ }^{1 \dagger}$, Bingbing $W u^{1 \dagger}$, Renchao Liu ${ }^{1}$, Yulan Lu ${ }^{1}$, Ping Zhang ${ }^{1}$, Caihong Shao ${ }^{2}$, \\ Ying Huang ${ }^{2 *}$ and Huijun Wang ${ }^{1 *}$ \\ ${ }^{1}$ Center for Molecular Medicine, National Children's Medical Center, Pediatrics Research Institute, Children's Hospital of \\ Fudan University, Shanghai, China, ${ }^{2}$ Department of Gastroenterology, Pediatric Inflammatory Bowel Disease Research \\ Center, National Children's Medical Center, Children's Hospital of Fudan University, Shanghai, China
}

Congenital diarrhea diseases are a heterogeneous group of conditions and are the major cause of neonatal mortality worldwide. Proprotein convertase 1/3 (PC1/3) deficiency has been associated with severe malabsorptive diarrhea, obesity, and certain endocrine abnormalities. We report an infant born to non-consanguineous parents who is diagnosed with $\mathrm{PC} 1 / 3$ deficiency due to nonsense homozygous variant (c.238 $\mathrm{C}>\mathrm{T}$, p.Arg80Ter) in the PCSK1 gene, identified by Trio-exome sequencing (Trio-ES). The baby girl presented with recurrent diarrhea, transient liver dysfunction and hypoglycemia. Trio-ES showed complete maternal uniparental isodisomy (iUPD) of chromosome 5. Our finding provides accurate genetic counseling to this family and expands the clinical spectrum of iUPD with pathogenic variants causing recessive disease.

Keywords: diarrhea, PCSK1 gene, uniparental isodisomy, infant, genetics

\section{BACKGROUND}

Congenital diarrhea diseases (CDDs) are a heterogeneous group of conditions characterized by watery diarrhea and are the major cause of neonatal mortalities worldwide (Younis et al., 2020). The World Health Organization defines diarrhea as three or more loose or liquid stools per day or an increase in defecation frequency. Early diagnosis of CDD is critical to prevent the progression of the disease to avoid adverse outcomes. With genetic testing increasingly applied to clinical use, the genetic causes of CDDs were gradually revealed and the candidate of causative genes was accumulating (Elkadri, 2020; Younis et al., 2020). These genes were functionally divided into five different subgroups, including the defects in epithelial nutrient and electrolyte transport, epithelial enzyme and metabolism, epithelial trafficking and polarity, enteroendocrine cell, and immune system (Thiagarajah et al., 2018; Elkadri, 2020).

Currently, four genes are reported to be related to enteroendocrine cell dysfunction, including NEUROGS (causing congenital malabsorptive diarrhea 4), PCSK1 (causing obesity with impaired prohormone processing/proprotein convertase 1/3 deficiency, or PCSK1 deficiency) (Younis et al., 2020), and genes causing a syndromic disease with diarrhea-ARX (leading to X-linked lissencephaly 2 or hydranencephaly with abnormal genitalia) and RFX (leading to Mitchell-Riley 
syndrome) (Thiagarajah et al., 2018; Elkadri, 2020). Among the above disorders, PCSK1 deficiency is an age-related CDD, with neonatal-onset severe malabsorptive diarrhea, but the intestinal phenotype will disappear later and the patient would even develop obesity with multiple endocrine abnormalities (Jackson et al., 1997; Frank et al., 2013; Pepin et al., 2019).

Uniparental isodisomy (iUPD), which means both alleles originating from a single-parental homologous chromosome, is an underestimated cause of autosomal recessive disorders (Yamazawa et al., 2010). Currently, nine patients had been reported with uniparental disomy of chromosome 5 [UPD(5)] with homozygous variants (Park et al., 2019; Gonzalez-Quintana et al., 2020). However, maternal iUPD of chromosome 5 (iUPD(5) mat) with a homozygous pathogenic variant in PCSK1 has never been reported.

Here, we report the first case of $\operatorname{iUPD}(5)$ mat leading to a homozygous nonsense variant in PCSK1 identified by TrioES. Clinicians and genetic counselors should be aware that for offspring of non-consanguineous parents, autosomal recessive disorders due to homozygous variants can occur as a result of uniparental isodisomy.

\section{CASE PRESENTATION}

The proband was a 7 month-23-day old girl at admission. She was born to a healthy non-consanguineous couple after an uneventful full-term pregnancy and was born without anomalies. But on day 3, the infant began diarrhea with watery stool over ten times a day. When she was 1 month old, she manifested jaundice and cholestasis with mild abnormal liver enzymes. During the first month, different types of infant formulas and breast milk were performed on her, but the diarrhea was not controlled. Then she was fed with amino acid-based formula until 8 months old, with diarrhea persisting. At her 8 months old, the routine stool test of her was not very informative, except for some fat globules existing. The enteric adenovirus, rotavirus, and norovirus were all negative tested by the immune gold standard method. The immunoglobulin, including IgA, IgG, IgM and IgE were normal, meaning that she had no immunity system deficiency. Her liver enzymes were still abnormal, AST 85.6 (normal 13-35 U/L); ALT 129.2 (normal 7-40 U/L), together with asymptomatic hypoglycemia, blood glucose 3.8 (normal 3.9-6.1 mmol/L). However, when she was 10 months old, her liver enzyme and blood glucose turned to normal. From then on, rice porridge was added to her meals with regular infant formula, and she can drink 300-500 $\mathrm{ml}$ water and 1,000 ml milk per day. Now she had loose stool twice to five times per day. The thriving records were shown in Figure 1A.

She got the physical examination five times. The height and weight were $50 \mathrm{~cm}$ and $3.3 \mathrm{~kg}$ at birth, $60.2 \mathrm{~cm}$ and $5.5 \mathrm{~kg}$ at 3 months old, $69 \mathrm{~cm}$ and $7.7 \mathrm{~kg}$ at 6 months old, $72 \mathrm{~cm}$ and $8.5 \mathrm{~kg}$ at 8 months old, $75 \mathrm{~cm}$ and $10 \mathrm{~kg}$ at 11 months old. The height and body mass index (BMI) score of the proband from birth to age 11 months was shown in Figure 1B. The BMI score increased gradually during the past 11 months. Her mother and father's height, BMI were $160 \mathrm{~cm}, 25.4 \mathrm{~kg} / \mathrm{m}^{2}$ and $175 \mathrm{~cm}, 37.5 \mathrm{~kg} / \mathrm{m}^{2}$, respectively. Pretest counseling was performed by the physician. Informed consent was signed by the parents. The criteria of genetic testing received approval from the ethics committees of the Children's Hospital, Fudan University (2015-130).

Molecular genetic analysis was ordered when the girl was 7 month-23-day old. Genomic DNA was extracted from the infant's and her parents' peripheral blood using a whole blood genomic DNA extraction kit (Qiagen, German). DNA fragments were enriched using the Agilent SureSelect XT Human All Exon $50 \mathrm{Mb}$ kit (Santa Clara, CA). Then DNA libraries were sequenced on the HiSeq2000/2500 sequencer according to the manufacturer's instructions (Illumina, San Diego, CA). The data analysis method followed the pipeline established in-house (Yang et al., 2019). The criteria of the molecular diagnosis followed the American College of Medical Genetics (ACMG) guidelines. We classified the variant to be a pathogenic variant, according to PVS1+PM2+PP4. UPD was detected using "B Allele Frequency" (BAF) (van Riet et al., 2018). The distribution of variant heterozygosity on each chromosome was calculated to scan the UPD event. The nonsense variant of PCSK1 was confirmed by Sanger sequencing using ABI 3,730 Genetic Analyzer (Applied Biosystems). Agilent SurePrint G3 comparative genomic hybridization (CGH) and SNP $4 \times 180 \mathrm{~K}$ microarray (Agilent Technologies, USA) was used to confirm the UPD(5) following the manufacturer's instructions. We used Agilent Cytogenomics software package for $\mathrm{CNVs}$ and the absence of heterozygosity $(\mathrm{AOH})$ calling and visualization.

Trio-ES data showed that $97.9 \%(1168 / 1193)$ variants had a BAF higher than 0.95 on chromosome 5 , meaning the absence of heterozygosity $(\mathrm{AOH})$, which is inherited from the mother (Figure 2A). Array-CGH showed no copy number variants in the proband but complete $\mathrm{UPD}(5)$ by showing $\mathrm{AOH}$ across the entire chromosome (Figure 2B). The sizes of AOH in this patient was $172.48 \mathrm{Mb}$ detected by array-CGH, and $170.95 \mathrm{Mb}$ detected by Trio-ES, respectively. Meanwhile, a homozygous nonsense variant in PCSK1 (NM_000439: c.238C > T, p.Arg80Ter) was detected in the proband, while her mother was a heterozygous carrier and her father was normal (Figure 2C). This variant was confirmed by Sanger sequencing (Figure 2D). The variant whose site is highly conserved among different species caused a protein truncation (Figure 2E). Trio-ES did not identify any other pathogenic or likely pathogenic variants associated with the patient's clinical features or other inherited diseases.

Combining the clinical phenotype (continuous neonatal diarrhea, BMI increase) and the molecular genetic finding, the proband was diagnosed with congenital proprotein convertase $1 / 3$ deficiency caused by a PCSK1 homozygous pathogenic variant due to $\mathrm{iUPD}(5)$ mat.

\section{DISCUSSION}

PCSK1 encodes the proprotein convertase subtilisin/kexin type 1, which involves the proteolytic activation of polypeptide hormones and neuropeptides precursors. Defects in this gene have been associated with susceptibility to obesity (RamosMolina et al., 2016) and congenital proprotein convertase 1/3 
A

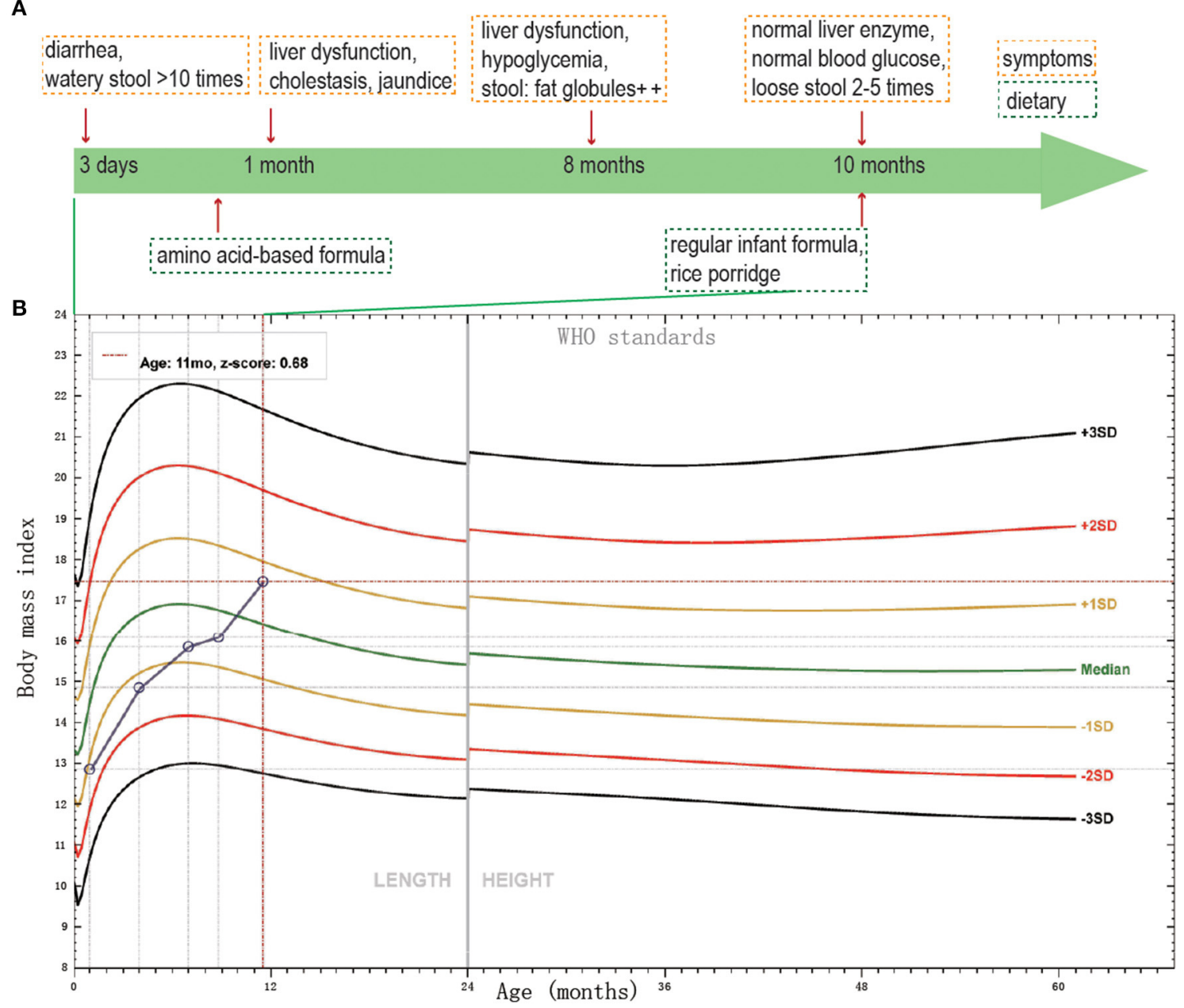

FIGURE 1 | The thriving records of clinical features of the patient. (A) The clinical symptoms and dietary of the patient. (B) BMI SD score of the proband, according to WHO standards, from birth to age 11 months.

deficiency (PC1/3 deficiency) (Martin et al., 2013; Pepin et al., 2019) -also called obesity with impaired prohormone processing [OMIM: 600955]. Here, we report a new case that suffered from recurrent diarrhea since 3 days old, with a homozygous nonsense pathogenic variant in PCSK1 (Arg80Ter). The variant was predicted to lead to a premature stop codon. The truncated protein may lose the entire catalytic domain, $\mathrm{P}$ domain, Cterminal tail, and part propeptide. This variant was once detected in a family with obesity disorder. The variant was overexpressed in the 293T cell line, and they found that the activity of wildtype PC1/3 was inhibited (Philippe et al., 2015). However, that is an experiment not with tissue/cells from the patient. In addition, truncating protein could be generated when some mRNA escapes nonsense-mediated decay (NMD). Therefore, the detection of truncating protein in a cell line does not rule out the possibility of NMD. Further studies will be needed to determine whether NMD occurs for this variant. Besides, all of the heterozygous carriers in the family showed obesity or overweight; the lowest BMI of the carrier was 26.91. The patient's mother in our study show overweight (BMI: 25.4) but not obesity.

We updated the summary of patients with PC1/3 deficiency carrying PCSK1 variants from Lucie Pe'pin's review (Pepin et al., 2019). The first patient carrying compound heterozygous variants of PCSK1 gene showing postnatal malabsorptive diarrhea and early-onset obesity was reported in 1997 (Jackson et al., 1997). Since then, 26 more cases of PC1/3 deficiency have been reported. Including our study, the 28 cases shared 25 distinct PCSK1 gene variants (Pepin et al., 2019; Serra et al., 2020) (Figure 3). All of the 28 patients had early-onset severe malabsorptive diarrhea, and one of them was firstly diagnosed with inflammatory bowel disease (Serra et al., 2020). Among them, 96.3 percent $(n=26 / 27)$ are younger than 1 month (seven patients under 3 days old). Early-onset obesity (before 5 years) was observed in $80 \%(n=20 / 25)$. Most of the patients got the genetic diagnosis when they suffered severe obesity and diabetes, or central diabetes insipidus. Our case was diagnosed at 8 months old. She was continually followed-up until 11 months; she could 


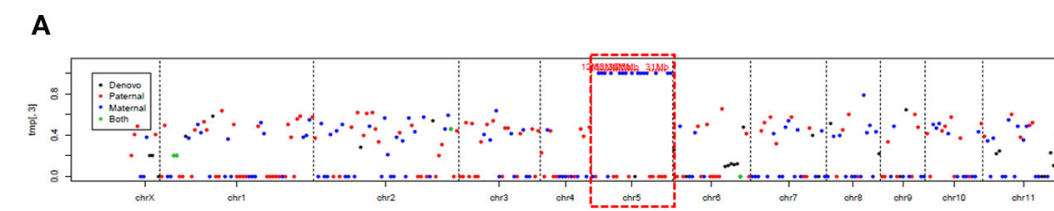

B

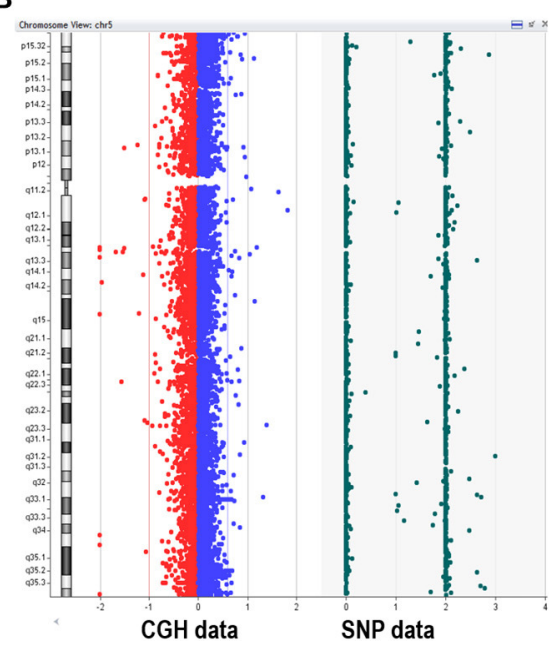

C

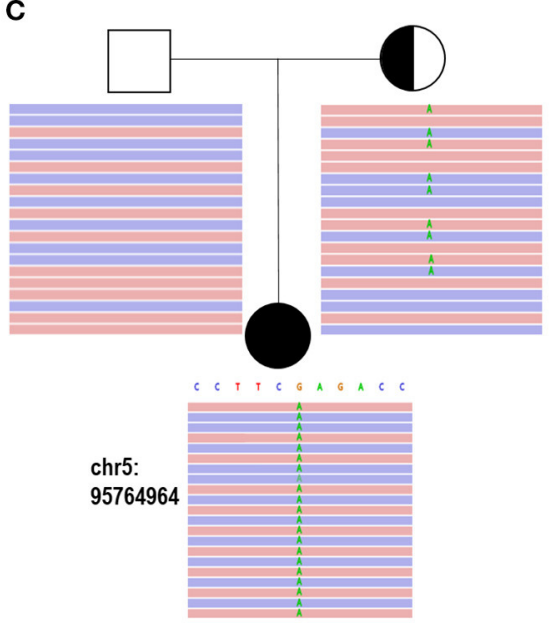

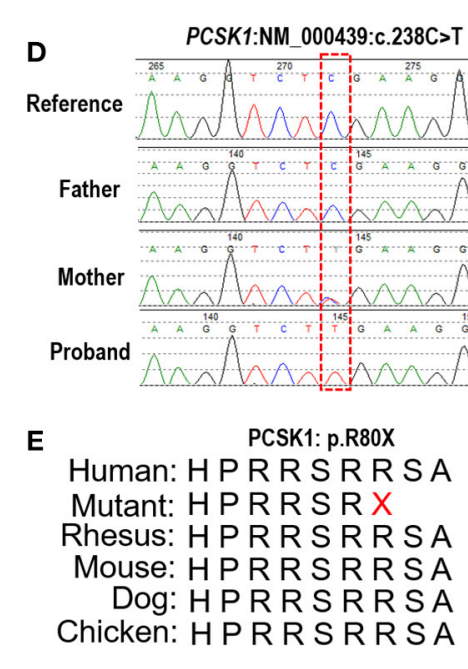

FIGURE 2 | The genetic test results of the patient. (A) Trio-ES identified homozygous variants on chromosome 5 and inherited from her mother, suggesting that the proband has UPD(5)mat. (B) Array-CGH confirmed UPD(5) by showing the copy neutral AOH across the whole chromosome 5. (C) A homozygous nonsense variant on PCSK1 (c.238C>T) is detected in the proband. Her mother is heterozygous and her father is normal. (D) The variant is confirmed by Sanger sequencing. (E) The variant site is conserved in different species.

\section{PCSK1: \\ NM_000439 \\ NP_000430}

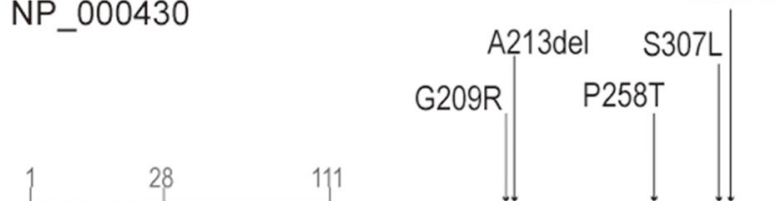

N309K

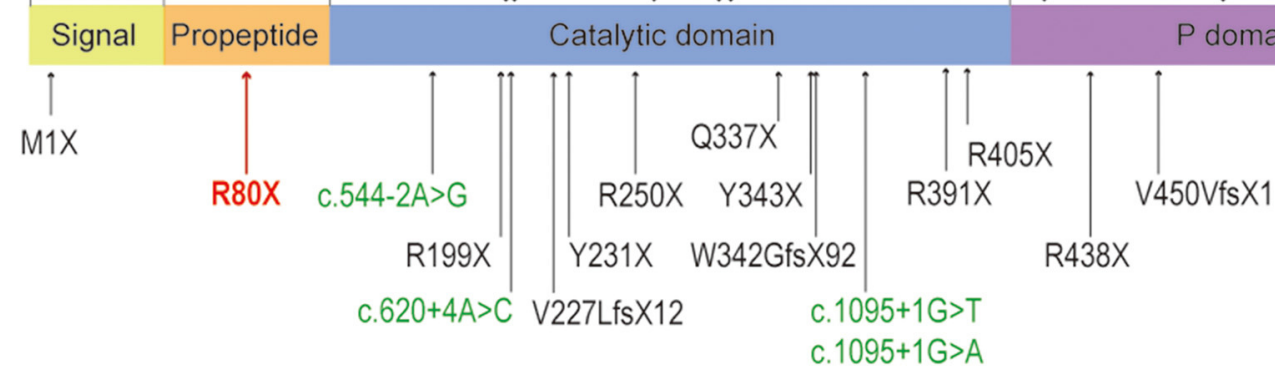

FIGURE 3 | The structure of PCSK1, and the reported variants caused diarrhea, including this study. The missense variants and non-frameshift variants were shown above the panel, the nonsense, frameshift variants or splice sites were shown below the panel. The splice sites were shown in green, the nonsense variant identified in this study was shown in red. Revised from reference (Martin et al., 2013).

drink milk/water $>1000 \mathrm{ml} /$ day. She has not presented obesity or diabetes, but her BMI was increasing. So, diet control was suggested to prevent obesity and to control glucose levels.

From a molecular diagnose viewpoint, 72 and 20 percent of PCSK1 pathogenic variants were located in the region coding for the catalytic domain and $\mathrm{P}$ domain, respectively. Sixty-eight percent of variants were deleterious, including 10 nonsense variants, 3 frameshift variants and 4 splice sites variants (Figure 3). However, there was no apparent genotype-phenotype correlation between individuals (Pepin et al., 2019). 
As reported, 88.5 percent (23 of 26) families had a history of consanguinity (Martin et al., 2013; Pepin et al., 2019). Among the homozygous pathogenic variants, only one patient (Asn423Lys) was not in consanguinity but inherited from both parents (Martin et al., 2013). In this study, our patient had the homozygous variant inherited from the heterozygous carrier mother. Both array-CGH and Trio-ES data exclude any genomic deletion in this infant. Using the method to detect UPDs from Trio-ES data in our team (Zhang et al., 2020, 2021), we found that variants of the infant on chromosome 5 were $97.9 \%$ homozygous and maternally inherited, suggesting it was $\mathrm{iUPD}(5)$ mat. It is worth mentioning that there are three imprinting genes- RHOBTB3, ERAP2, and VTRNA2-1 on chromosome 5. All of these regions were paternally imprinted, suggesting that imprinting is not a pathogenic mechanism for our patient. Till now, the reports of uniparental disomy of chromosome 5 due to homozygous mutant expression of recessive diseases are still rare. Our patient was the tenth case of $\operatorname{UPD}(5)$, and the fourth of UPD(5)mat. Different from the typical autosomal recessive disease, the recurrence risk for this family is relatively low.

In conclusion, we report the first case of $\mathrm{PC} 1 / 3$ deficiency caused by iUPD(5)mat with a nonsense homozygous variant of the PCSK1 gene. Our results highlight that patients, particularly in those families without consanguinity, have the possibility of UPD as a cause of autosomal recessive disorders. The early

\section{REFERENCES}

Elkadri, A. A. (2020). Congenital diarrheal syndromes. Clin. Perinatol. 47, 87-104. doi: 10.1016/j.clp.2019.10.010

Frank, G. R., Fox, J., Candela, N., Jovanovic, Z., Bochukova, E., Levine, J., et al. (2013). Severe obesity and diabetes insipidus in a patient with PCSK1 deficiency. Mol. Genet. Metab. 110, 191-194. doi: 10.1016/j.ymgme.2013.04.005

Gonzalez-Quintana, A., Trujillo-Tiebas, M. J., Fernandez-Perrone, A. L., Blazquez, A., Lucia, A., Moran, M., et al. (2020). Uniparental isodisomy as a cause of mitochondrial complex I respiratory chain disorder due to a novel splicing NDUFS4 mutation. Mol. Genet. Metab. 131, 341-348. doi: 10.1016/j.ymgme.2020.10.008

Jackson, R. S., Creemers, J. W., Ohagi, S., Raffin-Sanson, M. L., Sanders, L., Montague, C. T., et al. (1997). Obesity and impaired prohormone processing associated with mutations in the human prohormone convertase 1 gene. Nat. Genet. 16, 303-306.

Martin, M. G., Lindberg, I., Solorzano-Vargas, R. S., Wang, J., Avitzur, Y., Bandsma, R., et al. (2013). Congenital proprotein convertase $1 / 3$ deficiency causes malabsorptive diarrhea and other endocrinopathies in a pediatric cohort. Gastroenterology 145, 138-148. doi: 10.1053/j.gastro.2013.03.048

Park, G. Y., Jang, D. H., Lee, D. W., Jang, J. H., and Joo, J. (2019). Hereditary sensory and autonomic neuropathy $2 \mathrm{~B}$ caused by a Novel RETREG1 mutation (c.765dupT) and paternal uniparental isodisomy of chromosome 5. Front. Genet. 10:1085. doi: 10.3389/fgene.2019.01085

Pepin, L., Colin, E., Tessarech, M., Rouleau, S., Bouhours-Nouet, N., Bonneau, D., et al. (2019). A new case of PCSK1 pathogenic variant with congenital proprotein convertase 1/3 deficiency and literature review. J. Clin. Endocrinol. Metab. 104, 985-993. doi: 10.1210/jc.2018-01854

Philippe, J., Stijnen, P., Meyre, D., De Graeve, F., Thuillier, D., Delplanque, J., et al. (2015). A nonsense loss-of-function mutation in PCSK1 contributes to dominantly inherited human obesity. Int. J. Obes. 39, 295-302. doi: $10.1038 /$ ijo.2014.96

Ramos-Molina, B., Martin, M. G., and Lindberg, I. (2016). PCSK1 variants and human obesity. Prog. Mol. Biol. Transl. Sci. 140, 47-74. doi: 10.1016/bs.pmbts.2015.12.001

Serra, E. G., Schwerd, T., Moutsianas, L., Cavounidis, A., Fachal, L., Pandey, S., et al. (2020). Somatic mosaicism and common genetic variation contribute to genetic diagnosis for this girl may avoid unnecessary tests and provided precision treatment in time.

\section{DATA AVAILABILITY STATEMENT}

The datasets presented in this study can be found in online repositories. The names of the repository/repositories and accession number(s) can be found at: https://www.ncbi.nlm.nih. gov/, SUB8931511.

\section{ETHICS STATEMENT}

The studies involving human participants were reviewed and approved by CHFU Ethics Committee. Written informed consent to participate in this study was provided by the participants' legal guardian/next of kin. Written informed consent was obtained from the minor(s)' legal guardian/next of kin for the publication of any potentially identifiable images or data included in this article.

\section{AUTHOR CONTRIBUTIONS}

HW, YH, CS, and BW conceived and supervised the project. RL, $\mathrm{YL}$, and PZ contributed to data acquisition and analysis. YQ and $\mathrm{HW}$ wrote the manuscript. All authors approved the manuscript.

the risk of very-early-onset inflammatory bowel disease. Nat. Commun. 11:995. doi: 10.1038/s41467-019-14275-y

Thiagarajah, J. R., Kamin, D. S., Acra, S., Goldsmith, J. D., Roland, J. T., Lencer, W. I., et al. (2018). Advances in evaluation of chronic diarrhea in infants. Gastroenterology 154:e2046. doi: 10.1053/j.gastro.2018.03.067

van Riet, J., Krol, N. M. G., Atmodimedjo, P. N., Brosens, E., van IJcken, W. F. J., Jansen, M., et al. (2018). SNPitty: an intuitive web application for interactive Ballele frequency and copy number visualization of next-generation sequencing data. J. Mol. Diagn. 20, 166-176. doi: 10.1016/j.jmoldx.2017.11.011

Yamazawa, K., Ogata, T., and Ferguson-Smith, A. C. (2010). Uniparental disomy and human disease: an overview. Am. J. Med. Genet. C Semin. Med. Genet. 154C, 329-334. doi: 10.1002/ajmg.c.30270

Yang, L., Kong, Y., Dong, X., Hu, L., Lin, Y., Chen, X., et al. (2019). Clinical and genetic spectrum of a large cohort of children with epilepsy in China. Genet. Med. 21, 564-571. doi: 10.1038/s41436-018-0091-8

Younis, M., Rastogi, R., Chugh, A., Rastogi, S., and Aly, H. (2020). Congenital diarrheal diseases. Clin. Perinatol. 47, 301-321. doi: 10.1016/j.clp.2020.02.007

Zhang, P., Wu, B., Lu, Y., Ni, Q., Liu, R., Zhou, W., et al. (2020). First maternal uniparental disomy for chromosome 2 with PREPL novel frameshift mutation of congenital myasthenic syndrome 22 in an infant. Mol. Genet. Genomic Med. 8:e1144. doi: 10.1002/mgg3.1144

Zhang, P., Ying, W., Wu, B., Liu, R., Wang, H., Wang, X., et al. (2021). Complete IFN-gammaR1 deficiency in a boy due to UPD(6)mat with IFNGR1 novel splicing variant. J. Clin. Immunol. 41, 834-836. doi: 10.1007/s10875-02100970-3

Conflict of Interest: The authors declare that the research was conducted in the absence of any commercial or financial relationships that could be construed as a potential conflict of interest.

Copyright (C) 2021 Qian, Wu, Liu, Lu, Zhang, Shao, Huang and Wang. This is an open-access article distributed under the terms of the Creative Commons Attribution License (CC BY). The use, distribution or reproduction in other forums is permitted, provided the original author(s) and the copyright owner(s) are credited and that the original publication in this journal is cited, in accordance with accepted academic practice. No use, distribution or reproduction is permitted which does not comply with these terms. 